\title{
EL USO DE TÉCNICAS CUALITATIVAS EN LA EVALUACIÓN DE PROGRAMAS Los programas de desarrollo regional financiados por la Unión Europea
}

\author{
Verónica E. Viñas
}

Universidad Carlos III de Madrid

\section{RESUMEN}

En el año 2001 nos encontramos en un momento de transición de los fondos europeos destinados a la política de desarrollo regional, ya que - finalizado el período de programación en 1999 - se ha legislado nuevamente, pero manteniendo el objetivo general de cohesión económica y social dentro de una Unión Europea cada vez más diversa. A lo largo de 2000 y 2001 se han ido aprobando los programas concretos para cada una de las regiones de la Unión Europea.

En esta nueva programación se observa, estudiando los reglamentos, que existe cada vez un mayor interés de la Comisión Europea por conocer los impactos de las actuaciones financiadas con fondos europeos. De hecho, la normativa comunitaria referida al seguimiento y la evaluación ha sufrido cambios importantes entre la vigente hasta 1999 y la que se aplica a partir del año 2000. Los principales cambios se refieren a la mayor precisión en las referencias a la necesidad y obligatoriedad del seguimiento y de la evaluación, mejorando la concreción de sus objetivos; al aumento del grado de implicación y de las responsabilidades de los Estados miembros, y a la vinculación de la calidad y los resultados de las evaluaciones con repercusiones financieras para los Estados o regiones. El principal énfasis está puesto en la necesidad y pertinencia de realizar el seguimiento y evaluaciones de las intervenciones estructurales para conocer con mayor precisión su eficacia.

A la vista de las evaluaciones realizadas hasta la actualidad, ¿cuáles son los elementos que han permitido conocer en mayor grado las consecuencias (efectos e impactos) sobre la población y las regiones de las políticas de desarrollo regional? ¿Cómo se puede mejorar este conocimiento? Sobre estas cuestiones se reflexiona en este trabajo, argumentando que dos líneas de acción clave para la calidad de las evaluaciones son: la utilización del enfoque cualitativo en las evaluaciones, y el diseño y utilización de un sistema de seguimiento continuo de las actuaciones financiadas con fondos europeos. 


\section{DESARROLLO REGIONAL EN EL ÁMBITO DE LA UNIÓN EUROPEA}

En 1957 se constituyó la Comunidad Europea con la firma del Tratado de Roma. En él se señalaba la necesidad de garantizar un desarrollo armónico, pero no se diseñó una política concreta que tuviera este objetivo, aparte de la eliminación de las barreras arancelarias y de la apertura paulatina de los mercados. En 1975, coincidiendo con las primeras fases de reconversión industrial, se creó el Fondo Europeo de Desarrollo Regional (FEDER) y el Comité de Política Regional. Se complementaban, de esta forma, otros dos fondos europeos ya existentes desde los primeros años de la década de los sesenta: el Fondo Social Europeo (FSE) y el Fondo Europeo de Orientación y Garantía Agraria (FEOGA) (creados en 1960 y 1962, respectivamente). Sin embargo, hubo que esperar hasta la ampliación de la Comunidad a países más pobres, como Grecia, Portugal y España, en los años ochenta, para que se afirmaran la necesidad económica y la voluntad política de reducir las disparidades de desarrollo y de niveles de vida, reflejadas en los niveles de renta per cápita, en los niveles de productividad y en las tasas de desempleo. Prueba de ello es que a partir de la reforma de los Fondos Estructurales en 1988 (Comisión Europea, 1988) se duplicaron los fondos para las regiones comunitarias con un retraso estructural en su desarrollo y - desde entonces- no han dejado de crecer los presupuestos europeos asignados a la política de cohesión. En la actualidad, la cohesión económica y social figura en el Tratado de Maastricht (Unión Europea, 1992) como una de las prioridades políticas de la Unión Europea (al mismo nivel que la unión monetaria) y en la llamada Agenda $2000^{1}$, siendo la segunda política en importancia dentro de la Unión Europea en términos presupuestarios, después de la política agrícola. En concordancia con el establecimiento de estas prioridades políticas, en 1994 se aprobó el Fondo de Cohesión, un instrumento financiero de cohesión que aporta contribuciones económicas a los cuatro Estados miembros cuyo Producto Interior Bruto (PIB) por habitante se sitúa por debajo del 90 por 100 de la media comunitaria (España, Grecia, Irlanda y Portugal) para ser destinadas a proyectos relativos al medio ambiente y a redes transeuropeas de infraestructura de transporte (Comisión Europea, 1994). Es también después de la firma del Tratado de Maastricht cuando se crea otro instrumento financiero específico para la reconversión y modernización del sector pesquero (IFOP), en 1993.

De forma complementaria a los Fondos Estrtucturales, en 1989 se pusieron en marcha las Iniciativas Comunitarias, que se dirigen esencialmente a aspectos relacionados con los objetivos prioritarios de las medidas estructurales, con un interés comunitario específico, tales como la cooperación trans-

Con la adopción de una veintena de medidas legislativas, la Unión finalizó, en 1999, su proyecto titulado Agenda 2000. El proyecto se basó en una comunicación de la Comisión del año 1997: Agenda 2000: por una Unión más fuerte y más amplia (Comisión Europea, 1997). 
fronteriza, transnacional e interregional; el desarrollo rural; la lucha contra la discriminación en el mercado laboral; etc. (Mairal, 1994: 52-53).

La política de cohesión de la Unión tiene como objetivo corregir las disparidades de desarrollo, interviniendo en los factores económicos y sociales que las inducen y perpetúan. Por ello se la denomina "política estructural». No se trata únicamente de transferir dinero a las regiones pobres, sino de dotarlas de las condiciones necesarias para acelerar su desarrollo y mejorar su nivel de vida: dotación de equipamientos básicos, capacitación de la población activa, aumento de la capacidad tecnológica, creación de un entorno atractivo para las inversiones, etc.

La idea subyacente a esta política es que los desequilibrios de desarrollo no sólo perjudican a las regiones y a los colectivos menos favorecidos, sino que también entorpecen el crecimiento de la Unión en su conjunto, ya que impiden aprovechar al máximo el potencial económico, tecnológico y humano de que dispone la Unión Europea. A su vez, la demanda de bienes o servicios generada por las ayudas comunitarias redunda en beneficio de las empresas asentadas en los países y regiones más prósperos, pues les abre nuevas oportunidades de negocio.

Para España, la entrada en la Unión representó un gran reto en relación a la política regional. De hecho, antes de 1986, en el país no existía una política de desarrollo regional en concordancia con la importancia de los desequilibrios regionales internos, aunque sí medidas aisladas. Desde ese momento, la Administración central y las Comunidades Autónomas han tenido que adaptarse a las exigencias de la política regional comunitaria para poder beneficiarse de los Fondos Estructurales. La aportación financiera de los Fondos Estructurales a España se elevó a más de 1,967 billones de pesetas (precios de 1989) para el período transcurrido entre 1989 y 1993. La contribución se triplicó en el siguiente período (1994-1999), hasta una dotación superior a los 6,738 billones de pesetas (precios de 1994). Estas cuantías equivalen al 0,7 por 100 de la media anual del PIB durante el primer período y al 1,7 por 100 durante el segundo. Es decir, España recibió el 23 por 100 del presupuesto comunitario para Fondos Estructurales, sin contar con las ayudas procedentes de las Iniciativas Comunitarias y del Fondo de Cohesión. Para el período 2000-2006, España recibirá un porcentaje similar de estos fondos, es decir, más de 7,155 billones de pesetas. Para las diez Comunidades Autónomas pertenecientes al Objetivo 1 de la política regional comunitaria ${ }^{2}$, estos fondos representan un aporte financiero sustancial para sus presupuestos. Para otras, como Cataluña, País Vasco o Madrid, incluidas en el Objetivo $2^{3}$, lo importante no son tanto

${ }^{2}$ A partir de 2000 se incluyen dentro del Objetivo 1 las regiones con un PIB por persona inferior al 75 por 100 de la media comunitaria, al igual que en el período anterior, pero se agregan las regiones subvencionables en virtud del antiguo Objetivo 6 (Finlandia y Suecia) y las regiones ultraperiféricas (Departamentos franceses de Ultramar, Islas Canarias, Azores y Madeira).

3 A partir del año 2000 se incluyen dentro del Objetivo 2 no sólo las zonas industriales en declive (como hasta el año anterior), sino también las zonas rurales, las zonas urbanas con elevada 
los recursos transferidos «como la oportunidad de participar de pleno derecho en la arena comunitaria mediante los procedimientos de cooperación entre los distintos niveles de gobierno" (Morata, 1998: 386).

En el año 2001 nos encontramos en un momento de transición de los Fondos Estructurales, ya que - finalizado el período de programación en 1999- se ha legislado nuevamente sobre la política de desarrollo regional, pero manteniendo el objetivo general de cohesión económica y social dentro de una Unión Europea cada vez más diversa. Las reorientaciones acordadas se refieren tanto a los Fondos Estructurales (FSE, FEDER, FEOGA e IFOP) como al Fondo de Cohesión. Las nuevas condiciones generales aplicables a las intervenciones de los Fondos Estructurales se describen en un Reglamento horizontal (Comisión Europea, 1999k), que incluye disposiciones generales sobre los Fondos Estructurales. Según este Reglamento, la ayuda comunitaria se concentrará en lo sucesivo en tres objetivos prioritarios, en lugar de los seis objetivos anteriores ${ }^{4}$ :

- Desarrollo y ajuste estructural de las regiones menos desarrolladas (Objetivo 1).

- Reconversión económica y social de las regiones con deficiencias estructurales: industriales, rurales, urbanas y dependientes de la pesca (Objetivo 2$)$.

- Desarrollo de los recursos humanos (Objetivo 3).

La concentración también se ha aplicado a las Iniciativas Comunitarias, que pasan de trece a cuatro. Sólo se mantienen las iniciativas INTERREG (cooperación transfronteriza, transnacional e interregional), LEADER (desarrollo rural), EQUAL (lucha contra las discriminaciones y desigualdades en el acceso al mercado de trabajo) y URBAN (rehabilitación económica y social de las ciudades y de los barrios en crisis) ${ }^{5}$.

El Reglamento general sobre los Fondos Estructurales se completa con tres reglamentos más específicos que definen el objetivo que debe alcanzarse con cada uno de los fondos (FSE, FEDER e IFOP), así como los tipos de actuación que pueden beneficiarse de su intervención (Comisión Europea, 1999q, 1999 p y 1999 l, respectivamente). Las acciones del FEOGA estarán cubiertas,

tasa de desempleo de larga duración, elevado nivel de pobreza, medio ambiente deteriorado, criminalidad y delincuencia o bajo nivel de educación, y las zonas de pesca.

${ }^{4}$ Una comparación esquemática de los objetivos prioritarios de los Fondos Estructurales en el período 1994-1999 frente al período 2000-2006, incluyendo las referencias reglamentarias, se encuentra en Comisión Europea (2000 $f$ ).

5 Una comparación esquemática de las Iniciativas Comunitarias en el período 1994-1999 frente a las del período 2000-2006, incluyendo las referencias reglamentarias, se encuentra en Comisión Europea (2000f). Los comunicados de la Comisión a los Estados miembros fijando orientaciones para las iniciativas comunitarias URBAN, EQUAL, LEADER e INTERREG son del mes de abril del año 2000 (Comisión Europea, 2000a, 2000b, 2000c y 2000d, respectivamente). 
en lo sucesivo, por el nuevo reglamento sobre desarrollo rural (Comisión Europea, 1999j). El FEDER se centra en financiar las actuaciones dentro de los Objetivos 1 y 2, destinados a apoyar regiones y zonas definidas. El FSE se destina al conjunto de las acciones relacionadas con los recursos humanos (Objetivo 3) y no está regionalizado, ya que puede aplicarse al conjunto de las regiones y países de la Unión. Y el IFOP financia medidas complementarias de la política pesquera común, integradas en los programas de desarrollo regional con los otros fondos en las regiones del Objetivo 1, y con una financiación específica en las zonas situadas fuera de las regiones del Objetivo 1. El FEOGA, por su parte, se aplica en todo el territorio de la Unión Europea, la sección de Orientación y la sección de Garantía en las regiones subvencionables en virtud del Objetivo 1, y solamente la sección de Garantía en los demás sitios.

Al igual que los Fondos Estructurales, el Fondo de Cohesión seguirá siendo un pilar fundamental de la cohesión económica y social en la Unión durante el período 2000-2006. El objetivo principal del Fondo de Cohesión no varía con respecto al período anterior: financiar proyectos en los sectores del medio ambiente y de las redes transeuropeas de infraestructuras de transporte en beneficio de los Estados miembros con un PIB inferior al 90 por 100 de la media comunitaria. Por ello no se ha publicado un nuevo Reglamento, sino modificaciones al mismo (Comisión Europea, $1999 m$ y 1999n).

En la actualidad (principios de 2001) se están realizando las programaciones específicas por países y regiones (algunas ya han sido aprobadas por la Comisión Europea) que tendrán vigencia por siete años, hasta el 2006.

\section{LA EVALUACIÓN DE LA POLÍTICA REGIONAL}

Desde la reforma de los Fondos Estructurales de 1988 se observó que para la Unión Europea era cada vez más importante la evaluación de las políticas regionales, ya que se introdujo la obligatoriedad para la Comisión y los Estados miembros de evaluar las políticas estructurales europeas (Comisión Europea, 1988). Esto se ha corroborado con la reforma realizada en 1999 a partir de la Agenda 2000. No sólo es importante definir con corrección los problemas y necesidades de las regiones y diseñar programas que los corrijan, sino también establecer un sistema de evaluación que permita conocer de forma periódica - entre otras cuestiones - la eficacia, eficiencia, impacto, viabilidad, pertinencia y sostenibilidad de las actuaciones.

La normativa comunitaria referida al seguimiento y la evaluación ha sufrido cambios importantes entre la vigente hasta 1999 y la que se aplica a partir del año 2000. Los principales cambios son: a) la mayor precisión (y reforzamiento) de las referencias sobre la necesidad y obligatoriedad del seguimiento y de los tres tipos de evaluación (previa, intermedia y final); b) la determinación de quién deberá asumir la responsabilidad del seguimiento y de cada una de las evaluaciones; c) el aumento del grado de concreción de los objetivos del 
seguimiento y la evaluación; d) el aumento del grado de implicación y de las responsabilidades de los Estados miembros, y e) la vinculación de la calidad y los resultados de las evaluaciones con repercusiones financieras para los Estados (o regiones): la "reserva de eficacia»" ${ }^{6}$.

Por otro lado, la Comisión Europea - por iniciativa de sus diferentes Direcciones Generales - ha complementado la normativa comunitaria con numerosas directrices y documentos orientativos para la realización de las evaluaciones, explicando metodologías que pueden ser utilizadas y sugiriendo indicadores para cada una de las actividades que pueden ser financiadas por los fondos europeos ${ }^{7}$. Dentro de este esfuerzo de apoyar a los Estados miembros en la realización de las evaluaciones, la Unión Europea financió — para el período 1995-98 - un ambicioso programa, denominado MEANS ("Métodos de Evaluación de las Acciones de Naturaleza Estructural»), por el que se encargó a un grupo de expertos investigar, desarrollar y promocionar métodos y técnicas de evaluación de las intervenciones estructurales comunitarias. El objetivo era lograr una mayor coherencia y eficacia en los procedimientos utilizados para evaluar este tipo de actuaciones, ayudando a crear una cultura profesional y unos organismos profesionales, fomentando la discusión entre los agentes implicados, formulando marcos de referencia y difundiendo las prácticas ejemplares $^{8}$.

Pero... ¿qué se ha hecho hasta ahora para conocer los resultados de las actuaciones comunitarias englobadas en los programas de desarrollo regional?

Desde los años setenta se han llevado a cabo evaluaciones de algunas políticas regionales nacionales (sobre todo en el Reino Unido y, en menor medida, en los países nórdicos, Holanda e Irlanda) (Comisión Europea, 1999r: 141). Pero la reforma de los Fondos Estructurales, en 1988, impuso obligatoriamente las evaluaciones, y las introdujo de manera sistemática y generalizada. Por ejemplo, en España se han realizado entre los años 1999 y 2000 evaluaciones finales de las actuaciones cofinanciadas por todos los Fondos Estructurales y por algunas de las Iniciativas Comunitarias, al ser 1999 año de finalización de todas las programaciones.

Sin embargo, las numerosas evaluaciones (tanto las llevadas a cabo en estos

${ }^{6}$ A partir del año 2000, el 4 por 100 de los créditos previstos en cada reparto nacional será puesto en reserva al principio de cada período de programación. A mitad del período, la Comisión, de acuerdo con cada Estado miembro, asignará esta reserva a los programas considerados más eficaces. La eficacia se juzgará mediante indicadores previamente definidos, que reflejen la eficacia, la gestión y la ejecución financiera. Esto es lo que se ha dado en llamar «reserva de eficacia», tal como se dispone en el artículo 44 del Reglamento general (Comisión Europea, 1999k).

7 Por ejemplo, la Dirección General de Agricultura publicó las Orientaciones para la evaluación ex-post de los programas del Objetivo 5b. Periodo de programación 1994-1999 (Comisión Europea, 1999h), y la Dirección General de Política Regional y Cohesión, Coordinación y Evaluación de Operaciones publicó diversos documentos metodológicos (Comisión Europea, 1999f, $1999 g, 2000 e$ ).

8 Del programa MEANS han surgido diversas publicaciones, entre las que destacan los seis volúmenes publicados en 1999 (Comisión Europea, 1999c). 
últimos meses como las anteriores) presentan, en general, el siguiente problema: se describen las realizaciones físicas y financieras con gran detalle, pero se llega a conclusiones muy generales, en la mayoría de los casos de escasa utilidad para el apoyo a la toma de decisiones, uno de los objetivos principales (por no decir el principal) de toda evaluación. Por ejemplo, en un documento de la Unión Europea denominado «El impacto de los fondos estructurales en España» se afirma que los fondos europeos en el período de programación 19891993 en las zonas de declive industrial (Objetivo 2) contribuyeron «a la instalación de 235.000 líneas telefónicas digitalizadas y de $1.075 \mathrm{~km}$. de cable de fibra óptica, así como a la construcción o reparación de $3.000 \mathrm{~km}$. de carreteras locales». En otro documento, denominado «La Unión Europea, entre cohesión y disparidades», se afirma que "los programas de desarrollo regional cofinanciados por la Unión habrán dado origen a la construcción o rehabilitación de más de 17.000 kilómetros de grandes carreteras en las regiones menos desarrolladas». De estas afirmaciones y otras similares se deduce que las políticas de cohesión "han ejercido un notable efecto de palanca en las economías de los países y regiones beneficiarios, en particular entre las menos prósperas, a la vez que han contribuido a acrecentar el potencial económico del conjunto de la Unión»".

Es decir, salvo en contadas excepciones (aunque hay que resaltar que esas excepciones son cada vez más numerosas), se describen con detalle realizaciones (bienes y/o servicios producidos por las Administraciones públicas) pero no se realiza un análisis detallado de las consecuencias (resultados e impactos) de la utilización de esas realizaciones, sino que se mencionan cambios generales positivos atribuyéndolos a la existencia de los fondos. Se compara lo que ha ocurrido desde que existen los fondos europeos con lo que ocurría antes, estimando lo que hubiera ocurrido de haberse mantenido las tendencias observadas antes de recibir dichos fondos. Sin embargo, las estimaciones obtenidas realizando este tipo de análisis de las tendencias deben interpretarse con cautela, ya que no analizan directamente las repercusiones de la política estructural como tal, sino que, por implicación, atribuyen cualquier divergencia con respecto a las tendencias observadas anteriormente al efecto de las medidas aplicadas. Se supone, pues, que no cambian las tendencias observadas en el pasado y que no aparecen nuevos factores durante el período posterior a la existencia de los fondos — aparte de la introducción de la propia política europea- que afecten al resultado. Estos supuestos son, evidentemente, muy poderosos: es difícil identificar los resultados de cada programa y separarlos de los de otras acciones privadas, regionales o nacionales.

Pero esta situación no se debe a una falta de conocimientos de técnicas y

9 Documentos de la página web de la Comisión Europea: http://www.inforegio.cec.eu.int/ wbover. Se ha preferido citar ejemplos directamente de documentos de la Comisión y no de evaluaciones concretas porque en el texto nos estamos refiriendo a tendencias y características de las evaluaciones en general. 
métodos de investigación, sino que suelen ser otras las razones que la explican. Son diversas las causas que pueden aludirse, pero las principales son:

- Falta o escasez de información básica sobre las actuaciones y sobre las personas o territorios sobre los que se ha actuado. Esto se debe a que no existe — salvo en contadas excepciones - un sistema de seguimiento establecido y en funcionamiento, lo que obliga a los evaluadores a dedicar tiempo y esfuerzo a la mera descripción de las realizaciones.

- Amplio entramado institucional implicado. Es decir, existen numerosos gestores responsables de actuaciones cofinanciadas con fondos europeos. Y éstos no sólo están dispersos por un amplio número de servicios, direcciones generales y consejerías de las Administraciones regionales y centrales, sino que han ido en aumento desde la creación de los Fondos Estructurales.

- Diversidad de los territorios y grupos sociales destinatarios.

- Principio de adicionalidad de los Fondos Estructurales, que obliga a que todas las actuaciones realizadas con fondos europeos también tengan participación económica del Estado receptor (artículo 11 del Reglamento general sobre los Fondos Estructurales) (Comisión Europea, 1999k). Esto dificulta (por no decir que imposibilita) distinguir los resultados e impactos debidos a los fondos europeos de los que son consecuencia de las políticas no europeas.

- En algunos casos, los programas no son más que la agrupación de un conjunto de proyectos desconectados, cada uno con su propia lógica, sin un diagnóstico adecuado para justificarlos ni una definición previa de una estrategia de desarrollo (Comisión Europea, 1998: 3; Isla, Mella y Soy, 1998: 349).

- Escaso presupuesto disponible, en algunos casos, para la realización de las evaluaciones, si se compara con lo que se suele considerar adecuado destinar a este tipo de investigaciones en relación al monto de lo gastado en las actuaciones que quieren ser evaluadas.

- Escasa "cultura de la evaluación» en las Administraciones públicas, lo que conlleva ciertas resistencias y reticencias de los políticos y técnicos responsables de los programas a evaluar.

- Los equipos de evaluación de Fondos Estructurales suelen estar compuestos — sobre todo- por economistas e ingenieros, los que — por su formación académica básica- desconocen la forma de aplicar y utilizar técnicas no tradicionales de obtención de información, aunque conozcan su existencia e hipotética utilidad.

- El nivel de desarrollo de las estadísticas existentes en los países y en las regiones pone freno al seguimiento y la evaluación de los programas estructurales. Entre las lagunas observadas cabe citar, por su relevancia, la falta de una cobertura estadística circunscrita a las zonas afectadas y la ausencia de estadísticas sobre determinados aspectos (Comisión Europea, 1998: 5).

Estos problemas con que se han encontrado los evaluadores ha hecho subrayar la importancia de dos cuestiones: 
1) la utilidad de la evaluación cualitativa aplicada a las políticas de desarrollo regional, y

2) la importancia de contar con un sistema de seguimiento continuo en las Administraciones responsables de las actuaciones financiadas con Fondos Estructurales.

Estas dos cuestiones son las que se desarrollan a continuación.

\section{UTILIDAD DE LA EVALUACIÓN CUALITATIVA}

Es bien conocido por los profesionales dedicados a la evaluación que para realizar cualquier tipo de evaluación existen diferentes enfoques que pueden ser complementarios (cuantitativos y cualitativos), tanto para obtener información derivada de la ejecución del proyecto como de datos elaborados ad-hoc (Dunn, 1981: 38-40; Hogwood y Gunn, 1991: 171-207). Sin embargo, en las evaluaciones de Fondos Estructurales se han utilizado, principalmente, técnicas cuantitativas. Pero se observa en la actualidad una tendencia creciente a utilizar la perspectiva cualitativa, tanto de forma complementaria a la cuantitativa como de forma exclusiva. Esto se debe a diversas razones: a) el enfoque cualitativo es más adecuado que el cuantitativo para recoger información sobre actitudes, motivaciones, valores, creencias, aspiraciones o sentimientos; $b$ ) las técnicas cualitativas complementan a las cuantitativas, tanto si se realizan con anterioridad o con posterioridad a ella; $c$ ) el alto costo de los métodos cuantitativos en relación al costo total del proyecto (sobre todo en proyectos pequeños), y d) las técnicas cualitativas posibilitan la participación de la población en el diseño y análisis de las evaluaciones.

Son cuatro los métodos cualitativos que se han utilizado - principalmente- en la evaluación de programas de desarrollo regional: entrevistas a informadores individuales (o informantes clave), entrevistas a la comunidad, grupos de discusión y observación participante ${ }^{10}$. Pero este enfoque cualitativo no debe ser aplicado sin una reflexión previa. Esa reflexión debe incluir, al menos, los siguientes asuntos: 1) estudio de las dificultades de aplicación de los métodos cualitativos en programas de desarrollo regional; 2) análisis de las

${ }^{10}$ En este texto no se pretende exponer la utilización de cada una de esas técnicas, ni la forma en que se analizan, sintetizan y presentan los datos recogidos a través de ellas (algo que es frecuentemente olvidado en los textos que se dedican a la descripción de este tipo de técnicas), ya que excedería los límites de esta exposición. Existen numerosos textos sobre métodos cualitativos. Entre ellos, podemos destacar: Alonso (1994); Canales y Peinado (1994); Casley y Kumar (1990a); Ibáñez (1991); Ibáñez (1992); Kumar (1987); Kumar (1993); Taylor y Bogdan (1992); Valadez y Bamberger (1994); Viñas (1995); Ward, Bertrand y Brown (1991). Sobre el análisis, síntesis y presentación de los datos generados con técnicas cualitativas pueden consultarse: Alonso (1998); Argyle (1984); Aubel (1994); Bertrand, Brown y Ward (1992); Canales y Peinado (1994); Ibáñez (1992); Morris, Fitz-Gibbon y Freeman (1987); Patton (1987); Taylor y Bogdan (1992); Viñas (1995). 
ventajas y las desventajas de estos métodos para el análisis de esos programas, y 3) descripción de los tipos de datos y situaciones para los que se puede recomendar el enfoque cualitativo. Los apartados siguientes intentan contribuir a dicha reflexión, explicando las razones por las que se está tendiendo a utilizar con mayor frecuencia el enfoque cualitativo en la evaluación de programas de desarrollo regional.

\section{III.1. Producir información sobre actitudes, motivaciones, valores, creencias, aspiraciones o sentimientos}

Existen dos tipos de técnicas para la producción de información, las cuantitativas y las cualitativas. Cada una es más adecuada para diferente tipo de datos. Para la medición de cifras, tasas y porcentajes y para obtener información sobre hechos y acontecimientos, las técnicas más apropiadas son las cuantitativas. Pero para reunir información sobre actitudes, motivaciones, valores, creencias, aspiraciones o sentimientos, las técnicas más adecuadas son las cualitativas. O, en palabras de Alvira, "los datos cualitativos lo son en el sentido de recoger los significados e interpretaciones de los actores sociales, sus definiciones de la situación, marcos de referencia, etc. (...) tal como la comprenden y la entienden los actores sociales» (Alvira Martín, 1983: 65). Esto es así porque "las técnicas de investigación cuantitativas son objetivas, y las técnicas cualitativas de investigación son —en parte— subjetivas» (Ibáñez, 1985: 87). Los métodos cualitativos pueden utilizarse con carácter exploratorio, cuando el grado de conocimiento sobre el fenómeno es menor, y de forma complementaria a las técnicas cuantitativas.

En la encuesta estadística (la técnica cuantitativa de recogida de información más utilizada) la pertinencia de los temas a tratar es decidida por un observador externo, por lo que es un sistema de observación cerrado, pues las respuestas están contenidas en las preguntas. Además, generalmente el ámbito de respuestas permitido está predeterminado, ya que sólo es posible elegir una entre un conjunto cerrado de respuestas. El entrevistado no puede crear alternativas.

El enfoque cualitativo permite a los entrevistados — por un lado- expresar sus ideas de forma espontánea, sin ser estructuradas previamente por otras personas, y - por otro- posibilita el tratamiento de temas que no habían sido previstos en el diseño del trabajo. En los grupos de discusión, por ejemplo, los miembros del grupo generan nuevas ideas porque se estimulan entre ellos. La dinámica del grupo empuja a los participantes a responder a las opiniones, comentarios e ideas de los otros, surgiendo - de esta forma- nuevas líneas o perspectivas de análisis, que no hubieran sido identificadas con una encuesta estructurada. Las entrevistas abiertas o semiestructuradas, los grupos de discusión, las reuniones de comunidad, los estudios de caso, la observación participante, etc., son técnicas cualitativas de recogida de información. Todas ellas 
permiten recoger de forma más acertada la información que no había sido previamente pensada por la persona encuestada, ya que ésta no se encuentra forzada a elegir una, y sólo una, de las respuestas predeterminadas, gozando de más libertad de expresión.

Un ejemplo interesante ocurrió en una investigación en la India, cuando el investigador pidió a los habitantes de dos aldeas que definieran sus propias categorías y criterios de bienestar. A continuación se compararon esas definiciones con datos recogidos veinte años antes. Los 36 hogares cuyos ingresos se habían reducido en forma significativa en términos reales estaban, como promedio, mejor según los criterios que habían expresado ellos mismos. Si bien su ingreso real per cápita era menor, en su opinión, otras cuestiones habían mejorado su vida: mejor vivienda, uso habitual del calzado, menor dependencia de patrones y terratenientes, y menos necesidad de emigrar en busca de trabajo. Como se pone de manifiesto en esta y en otras investigaciones, las descripciones que hacen las propias personas del bienestar y de las privaciones (de la buena y la mala vida) tienden a ser multidimensionales (Chambers, 1995: 14-15).

Los proyectos de desarrollo se realizan para conseguir un impacto sobre la vida de un número considerable de personas. Estos proyectos se basan en un conjunto de supuestos explícitos o implícitos sobre las necesidades de la población, sobre la forma en que van a responder a ciertas actividades y sobre la manera en que sus vidas se verán afectadas (Danziger y Portney [eds.], 1988: 1-2). Debido a que muchos proyectos producen cambios importantes en las vidas de la población (y no siempre cambios positivos), los planificadores y los gestores deberían asumir la obligación de entender qué siente la población sobre esos proyectos y cómo se ve afectada por ellos, ya que hay que respetar el derecho fundamental de la gente a definir sus propias necesidades y a establecer su propio programa. Aunque los métodos cuantitativos pueden describir algunos de los cambios que ocurren, generalmente no revelan cómo la gente percibe esos cambios. En este caso, los métodos cualitativos resultan más adecuados (Valadez y Bamberger, 1994: 331).

\section{III.2. Complementar el enfoque cuantitativo}

El método cualitativo, además, puede utilizarse antes de diseñar el cuestionario de una encuesta, ya que sirve para identificar asuntos a incluir en el mismo y adecuar el texto del cuestionario al vocabulario utilizado por la población estudiada (Hedrick, 1994: 51). La encuesta permitirá conocer la importancia cuantitativa y la distribución y frecuencia en la población total de los resultados alcanzados con el trabajo cualitativo.

Por otro lado, la utilización de técnicas cualitativas con posterioridad a la realización de una encuesta también resulta de interés, ya que aquéllas contribuirán a una interpretación más profunda de los datos de la encuesta. 
Además, la información obtenida con un enfoque cualitativo puede usarse para comparar con la información de encuestas. Las encuestas se componen de un conjunto predeterminado de respuestas, que pueden no incluir la respuesta que el entrevistado daría si tuviera más opciones. Por otro lado, el entrevistado puede resistirse a confiar sus opiniones a un extraño, sobre todo en preguntas estructuradas. Esta resistencia puede tener su origen en factores culturales locales que impidan revelar ciertos datos a un extraño.

Uno de los problemas a la hora de la evaluación es que no todos los aspectos son medibles a través de la encuesta. De hecho, en muchos informes de evaluadores de proyectos de desarrollo rural se cuestiona la utilidad de las encuestas "porque no son los métodos más adecuados para obtener información", porque "no sirven para analizar los aspectos cualitativos del proyecto" (Ocampo, 1994), pero pocas veces se dan alternativas. La encuesta puede enfocarse a los aspectos productivos y a medir algunos efectos directos e indirectos del proyecto: ingresos, migración, empleo, tenencia de la tierra, etc. Sin embargo, para averiguar las fortalezas y debilidades del proyecto, cómo y por qué los miembros de la población objetivo se convierten en beneficiarios, cómo se relacionan entre sí los beneficiarios, cómo el proyecto es percibido por los beneficiarios, por los no beneficiarios y por los técnicos del proyecto, las técnicas cualitativas serán más adecuadas que las cuantitativas (Patton, 1987: 23).

La siguiente pregunta de una encuesta nos puede servir de ejemplo de la ambigüedad que la misma puede presentar y de las diferentes interpretaciones que el entrevistado puede otorgarle:

"La información que ha recibido de los formadores del Proyecto en los últimos cuatro años, ¿le parece adecuada a sus necesidades?»: Muy adecuada/Bastante adecuada/Poco adecuada/Nada adecuada/No sabe - no contesta.

Es muy probable que el entrevistado no sepa qué respuesta escoger de las que se le presentan, por considerar que la información recibida de los formadores es adecuada en algunos aspectos y en otros no, o le parezca más adecuada la que recibió durante el último año que la recibida anteriormente. Por esa razón, quizá se incline por la respuesta "bastante adecuada», pero con ella perderemos mucha de la riqueza de la percepción que esta persona tiene sobre el Proyecto. Con una técnica en que se permita al entrevistado expresar sus opiniones, percepciones, valores, etc., sin obligarlo a limitarse a respuestas previamente estructuradas, la información obtenida será mucho más detallada y rica en matices, aunque al mismo tiempo sea más difícil de sintetizar.

Lo anterior no significa que haya aspectos que puedan ser medidos y otros que no puedan serlo, ya que existe un «difuso y cambiante límite entre lo inmensurable y lo mensurable» (Feinstein, 1995), sino que para obtener una u otra información será más adecuado uno u otro enfoque. 


\section{III.3. Generar información pertinente y oportuna}

La evaluación de proyectos se realiza para proveer oportunamente información adecuada para ayudar en el proceso de toma de decisiones a los planificadores, gestores y ejecutores. Las técnicas cualitativas pueden generar información relevante con un costo (en tiempo y dinero) relativamente bajo. Por el contrario, las técnicas cuantitativas requieren una inversión en tiempo y recursos que no están a disposición de los gestores de los proyectos de desarrollo. De todas formas, aunque sí se contara con esos recursos, el largo proceso de recopilación, análisis y síntesis de una encuesta media o grande impediría obtener los resultados en el momento en que se necesitaran para la toma de decisiones (Casley y Kumar, 1990b: 1; Kumar, 1993 :9-12).

Muchas evaluaciones concluyen $\operatorname{tarde}^{11}$, y la información que se precisa para ayudar a los administradores a tomar decisiones se vuelve inútil — por muy precisa que sea- si se suministra después de haber pasado el momento de la toma de decisiones. Y esto impone — con frecuencia - restricciones muy riguroso a la (o las) persona(s) que está(n) realizando la evaluación.

\section{III.4. Permitir la participación de los beneficiarios en la evaluación}

Desde hace algunos años se ha empezado a considerar importante realizar evaluaciones participativas, es decir, evaluaciones en las que los beneficiarios no son meramente fuente de datos, sino que participan también en el diseño, realización y análisis de los datos de la evaluación. Esto se ha conseguido en diversos grados, pero - al menos — se ha extendido la idea de que tales intentos darán más calidad a las evaluaciones y permitirán a los evaluadores sacar conclusiones más pertinentes (Casley y Kumar, 1990a: 125). Por ejemplo, uno de los criterios de selección de las iniciativas comunitaria LEADER es la participación de la población. La participación de los beneficiarios en los proyectos (no sólo en la evaluación, sino también en la planificación y en la gestión) está relacionada con mayores probabilidades de sostenibilidad futura de las acciones de desarrollo. El problema suele ser que la población local no tiene los conocimientos y experiencia necesarios para realizar la planificación, ejecución y análisis de una evaluación. Por ello, las técnicas cualitativas son más apropiadas para realizar una evaluación participativa que las cuantitativas. El arte de conducir discusiones de grupo, por ejemplo, puede ser aprendido, e incluso algunas personas tienen cierto talento para ello, aunque sean iletradas, ya que las técnicas cualitativas se basan más en la discusión/conversación que en el análisis de los números (Uphoff, 1992: 9/13).

11 Este hecho es subrayado en los propios documentos de la Comisión, por ejemplo en Comisión Europea (1998: 2). 


\section{UN SISTEMA DE SEGUIMIENTO CONTINUO PARA APOYAR LAS EVALUACIONES}

Como ya se mencionó, la Comisión ha manifestado un interés creciente en el establecimiento de sistemas de seguimiento en las actividades financiadas con Fondos Estructurales, sobre todo a partir de la nueva reglamentación vigente desde el año 2000. Con anterioridad a esta fecha, no existía ninguna disposición específica que determinara las responsabilidades de los Estados miembros en relación al seguimiento de las actuaciones. Por otro lado, aunque se establecía la obligatoriedad de conformar un Comité de seguimiento, no se daba ninguna definición precisa de su papel. A partir de la última reforma de los Fondos Estructurales (vigente desde el año 2000) esta situación cambia, principalmente en tres aspectos: a) se especifica que el Estado miembro asume la responsabilidad general del seguimiento, detallando en qué consiste esa responsabilidad (recopilación y transmisión de datos, elaboración de un informe anual de ejecución, etc.); b) se especifican las responsabilidades del Comité de seguimiento, $\mathrm{y} c$ ) se definen de forma reglamentaria el objetivo y el contenido de los indicadores de seguimiento y el informe anual de ejecución ${ }^{12}$.

La idea que subyace a estas modificaciones es que, una vez definidas y puestas en marcha las actuaciones concretas dentro de la política estructural, es necesario y conveniente realizar el seguimiento y - posteriormente- la evaluación de las mismas.

El «seguimiento» es el examen periódico de la ejecución de un programa, para comprobar si la utilización de los recursos, los calendarios de trabajo, las realizaciones y los resultados esperados se están llevando a cabo de acuerdo con el plan trazado. Se basa en un sistema de información alimentado principalmente por los gestores, referido, sobre todo, a los objetivos operativos (realizaciones). Su objetivo principal es ayudar a los responsables de las actuaciones públicas en la toma de decisiones relativas a planificación y ejecución (Naciones Unidas, 1985: 18).

La «evaluación» es una investigación objetiva y sistemática para emitir juicios de valor sobre una intervención pública, interpretando y analizando con métodos rigurosos la información incluida en el sistema de seguimiento y otra información recogida y/o producida especialmente. Su misión es determinar la pertinencia, la eficacia, la eficiencia, el impacto, la calidad y la sostenibilidad de todas las actividades, para ayudar a mejorar las actuaciones que se hallan aún en marcha y en la planificación y ejecución de programas futuros.

La clasificación que utiliza la Comisión en sus documentos diferencia entre recursos utilizados, actividades realizadas, realizaciones (referidas a los bienes y/o servicios producidos), resultados (referidos a los efectos directos e inmediatos) e impactos (referidos a los efectos a largo plazo). Recoger información de forma continua de estos cinco elementos resulta poco factible en la realidad.

12 Véase Reglamento general, artículos 34, 35, 36, 37 y 46 (Comisión Europea, 1999k). 
De hecho, lo que en el ámbito del seguimiento y la evaluación suele recomendarse y considerarse adecuado es que los organismos gestores recopilen información relativa a los recursos, a las actividades, a las realizaciones y a algunos resultados de sus acciones, y que el grueso de la información sobre resultados y la información sobre impactos se produzca en evaluaciones periódicas, a través de estudios específicos de casos o muestras de los colectivos atendidos. La experiencia ha demostrado que si existe un sistema de seguimiento bien diseñado y que se alimenta de forma continua, las evaluaciones —que son investigaciones esporádicas - llegan a conclusiones y recomendaciones más útiles para la programación y la gestión. De lo contrario, los evaluadores tendrán que dedicar gran parte de su tiempo a realizar un trabajo de recopilación de información básica dispersa entre los organismos gestores, de difícil homogeneización y análisis. En los casos en que los organismos gestores de fondos europeos tienen un sistema de seguimiento continuo bien diseñado y actualizado, las conclusiones de las evaluaciones llevadas a cabo pueden centrarse en el estudio de los efectos e impactos concretos, resultando más útiles para ayudar a la toma de decisiones relativas a la planificación y ejecución de programas.

La necesidad de diseñar un sistema continuo de seguimiento de las acciones financiadas por fondos europeos tiene una doble justificación: la primera, de tipo normativo o jurídico; la segunda, de carácter económico-presupuestario (Comunidad de Madrid, 2000).

\section{IV.1. Desde el punto de vista reglamentario}

El decimocuarto «considerando» del Reglamento Marco de los Fondos Estructurales vigente en la etapa de programación anterior (Reglamento CEE núm. 2081/93) establecía: «(...) que conviene reforzar la apreciación previa, el seguimiento y la evaluación posterior y establecer una mayor flexibilidad en la ejecución de las intervenciones estructurales comunitarias a fin de responder a las necesidades reales; que, en aras de la eficacia, conviene proceder a una apreciación detallada antes de comprometer recursos comunitarios para garantizar que producirán ventajas socioeconómicas relacionadas con los recursos movilizados $(. .)$.$» .$

Esta voluntad de la Comisión recibió un nuevo impulso en los actuales Reglamentos reguladores del funcionamiento de los Fondos Estructurales (2000-2006). En concreto, el vigente Reglamento por el que se establecen disposiciones generales sobre los Fondos Estructurales (Reglamento CE núm. 1260/99) dispone en sus considerandos:

"(...) que una de las garantías de la eficacia de la acción de los Fondos Estructurales es un seguimiento eficaz; que es necesario mejorar el seguimiento (...)» (considerando 46). 
"(...) que es fundamental para el seguimiento que existan indicadores e informes anuales de ejecución y que es necesario definirlos mejor para que reflejen de manera fiable el grado de ejecución de las intervenciones y la calidad de la programación" (considerando 49).

El Reglamento desarrolla estos principios dedicando el Título IV a la «Eficacia de las intervenciones de los Fondos», con once detallados artículos referidos al seguimiento, a la evaluación y a la reserva de eficacia (Comisión Europea, $1999 k$ ). Estos artículos refuerzan, con mayor claridad aún, la opinión de la Comisión de que es necesario mejorar los sistemas de seguimiento y de evaluación actualmente existentes.

Las evaluaciones intermedias de los fondos europeos realizadas en 1997, y las evaluaciones finales llevadas a cabo entre los años 1999 y 2000, pusieron de manifiesto que el sistema de información y evaluación vigente no se encuentra adaptado a los requisitos solicitados por la Comisión, especialmente en tres aspectos: cuantificación de objetivos, generación de indicadores físicos sobre el desarrollo de las distintas acciones y evaluación de impactos derivados de dichas intervenciones.

\section{IV.2. Desde el punto de vista económico y presupuestario}

No obstante lo apuntado, la implantación de un sistema de seguimiento de las actuaciones desarrolladas por cualquier Administración pública no constituye tan sólo una obligación legal con posibles repercusiones económicas, sino también una oportunidad de cara a mejorar la gestión de las intervenciones dirigidas a impulsar los cambios estructurales en el tejido económico, social o territorial.

En buena medida, el desarrollo de un sistema racional y estructurado de información sobre la gestión de la política estructural europea debe generar necesariamente repercusiones significativas sobre la mejora de la calidad de la gestión pública, así como sobre la cantidad, calidad y transparencia de la información disponible sobre estas cuestiones, aspectos cada día más demandados, tanto desde el punto de vista político como social.

El seguimiento y evaluación de las acciones públicas son, en definitiva, una excelente vía para compatibilizar la creciente demanda social de bienes y servicios públicos con las restricciones existentes para el incremento del gasto público, ya que se trata de una herramienta de análisis y, sobre todo, de gestión, cuyo fin último estriba en suministrar información relevante, tanto a técnicos y gestores como a los órganos políticos en los que radica en última instancia la capacidad de decisión, para mejorar la asignación de unos recursos escasos, es decir, para aumentar la eficiencia del gasto público.

Por otro lado, un sistema de seguimiento continuo no sólo permite cumplir con la normativa y contribuir a mejorar la gestión, sino que también facili- 
ta el trabajo a todos los organismos públicos implicados en actuaciones financiadas por fondos europeos. Esto es así porque un sistema de seguimiento continuo pone a su disposición de forma rápida y actualizada la información necesaria para la elaboración de los informes semestrales y anuales que les exigen los organismos coordinadores de las políticas estructurales.

\section{EVALUACIÓN CUALITATIVA Y SISTEMA DE SEGUIMIENTO CONTINUO... ¿POR QUÉ Y PARA QUÉ?}

A la vista de lo apuntado hasta el momento, podríamos decir que a principios del año 2001 nos encontramos con una confluencia de situaciones que corroboran que para mejorar la eficacia, eficiencia y utilidad de las evaluaciones de programas de desarrollo regional financiados con fondos europeos son recomendables dos líneas de actuación: 1) la utilización del enfoque cualitativo en las evaluaciones de estas actuaciones, combinado con metodología cuantitativa en la medida que se considere pertinente, y 2) el diseño y utilización de un sistema de seguimiento continuo.

Las situaciones que han llevado a afirmar la importancia y relevancia de estos dos elementos, ya apuntados en apartados anteriores de este trabajo, se resumen de la siguiente forma:

- El creciente interés de la Comisión por conocer los impactos de las actuaciones financiadas con fondos europeos, reflejado en los diversos reglamentos que los regulan. De hecho, la normativa comunitaria referida al seguimiento y la evaluación ha sufrido cambios importantes entre la vigente hasta 1999 y la que se aplica a partir del año 2000. Los principales cambios son: a) la mayor precisión (y reforzamiento) de las referencias sobre la necesidad y obligatoriedad del seguimiento y de los tres tipos de evaluación (previa, intermedia y final); b) la precisión de quién deberá asumir la responsabilidad del seguimiento y de cada una de las evaluaciones (el Estado miembro o la Comisión); c) el aumento del grado de concreción de los objetivos del seguimiento y la evaluación; d) el aumento del grado de implicación y de las responsabilidades de los Estados miembros, y e) la vinculación de la calidad y los resultados de las evaluaciones con repercusiones financieras para los Estados (o regiones): la reserva de eficacia.

- La necesidad de mejorar la gestión de las intervenciones dirigidas a impulsar cambios estructurales, tanto por demandas políticas como sociales. Este objetivo puede conseguirse, en parte, con evaluaciones de calidad. De hecho, es significativo que un documento publicado por la Comisión tras la realización de las evaluaciones intermedias de los programas estructurales del período 1994-1999 se titule «Evaluación para una mejor gestión». De este texto pueden extraerse las siguientes afirmaciones significativas: "Las evaluaciones intermedias no son un fin en sí mismas, sino un medio para mejorar la 
calidad y pertinencia de la programación (...). Estas evaluaciones no se realizan con la simple finalidad de dar cumplimiento a las actuales normas comunitarias sobre programación, sino que también se han introducido para responder al creciente interés manifestado al respecto por las autoridades nacionales o regionales, para quienes la evaluación es cada vez más un medio de mejorar la gestión de los fondos y maximizar los resultados obtenidos. La evaluación intermedia permite determinar qué tipo de reorientaciones pueden ser las más convenientes para alcanzar los objetivos originales» (Comisión Europea, 1999b: 3).

- Las conclusiones de las evaluaciones intermedias y finales llevadas a cabo en los últimos años. Numerosas evaluaciones llevadas a cabo en los últimos años ponen de manifiesto que la mayoría de las Administraciones públicas responsables de actuaciones financiadas por fondos europeos no cumplen - en la actualidad - con las exigencias comunitarias en materia de calidad de los sistemas regionales y nacionales de información sobre los resultados físicos e impactos de las intervenciones.

De hecho, la aplicación de metodologías cualitativas combinadas con metodologías cuantitativas en las evaluaciones ha permitido no sólo describir realizaciones físicas, sino también describir las consecuencias directas sobre la población de esas realizaciones, distinguir entre los colectivos más y menos favorecidos por los programas y las razones que explican esas variaciones, detectar acciones innovadoras con mayor capacidad de impacto positivo, y establecer recomendaciones concretas para mejorar la gestión y la eficacia de las actuaciones.

Las evaluaciones llevadas a cabo con un enfoque cualitativo en España se han realizado entroncando con la amplia aplicación de la metodología cualitativa. De hecho, existe un amplio conocimiento de métodos cualitativos en España (Sarabia y Zarco, 1997), aplicados a investigaciones sociológicas en general, y al área de consumo y de estudios de mercado en particular. Dentro del campo de las evaluaciones de Fondos Estructurales, este tipo de enfoque se ha aplicado, sobre todo, en los programas sociales, pero con mucho menos frecuencia en las evaluaciones de otros sectores de las actuaciones públicas (agricultura, turismo, infraestructura, medio ambiente...). Esto se debe -entre otras razones - a que los equipos de evaluación están conformados, sobre todo, por economistas e ingenieros, quienes — por su formación básica- desconocen la mecánica de la aplicación del enfoque cualitativo, aunque conozcan su utilidad. En la actualidad no nos encontramos con un problema de opciones metodológicas a utilizar en las evaluaciones ${ }^{13}$, sino con los siguientes problemas: a) déficit en los conocimientos para aplicar un enfoque cualitativo

13 La complementariedad entre las perspectivas cuantitativa y cualitativa se ha abordado en numerosos textos. Cabe mencionar: Alvira Martín (1983); Conde (1987); Conde (1990); Delgado y Gutiérrez (coords.) (1994). 
dentro de los equipos responsables de las evaluaciones de Fondos Estructurales; b) poco reconocimiento y aceptación por parte de los clientes (las Administraciones públicas), y $c$ ) insuficiente información de base sobre realizaciones y sobre la situación de partida (la situación anterior a la aplicación de los programas). Por ello, sería recomendable que los profesionales de la evaluación utilicen con mayor frecuencia un enfoque cualitativo (complementando al enfoque cuantitativo), cuando sea pertinente, y que los responsables de los organismos gestores y coordinadores de los Fondos Estructurales conozcan y acepten su utilidad y rigurosidad metodológica. En relación a esta última cuestión, hay que subrayar que uno de los efectos positivos de los Fondos Estructurales europeos en España ha sido que la obligatoriedad de su evaluación ha creado, o ayudado a crear, en las diversas Administraciones públicas una cierta cultura de la evaluación. Es decir, que los responsables públicos y los gestores a cargo de actuaciones financiadas con fondos europeos han comenzado a comprender la utilidad del seguimiento y la evaluación, más allá de su obligatoriedad.

Por otro lado, el diseño y mantenimiento de un sistema de seguimiento ha permitido tener un conocimiento real y actualizado de las realizaciones que se llevan a cabo, evitando, así, que los evaluadores tengan que dedicar gran parte de su tiempo a la producción de datos descriptivos. De esta forma, han podido dedicarse al análisis, predicción y establecimiento de recomendaciones útiles para la mejora de la eficacia de los programas evaluados o para la planificación y gestión de programas futuros.

\section{BIBLIOGRAFÍA}

\section{a) Legislación y otros documentos de la Unión Europea}

Comisión Europea (1988), Reglamento (CEE) 2054/88 del Consejo, de 24 de junio de 1988, Reglamento Marco de la reforma de los Fondos Estructurales.

- (1994), Reglamento (CE) 1164/1994 del Consejo, de 16 de mayo de 1994, por el que se crea el Fondo de Cohesión, Diario Oficial de las Comunidades Europeas de 25 de mayo.

- (1997), Agenda 2000. Por una Unión más fuerte y más amplia, COM (97) 2000, presentada el 16 de julio.

- (1998), Informe especial núm. 15/98 sobre la evaluación de las intervenciones de los Fondos Estructurales para los períodos 1989-1993 y 1994-1999, acompañado de las respuestas de la Comisión, Diario Oficial de las Comunidades Europeas de 16 de noviembre.

- (1999a), Evaluación de los programas de desarrollo rural 2000-2006 financiados con cargo al Fondo Europeo de Orientación y Garantía Agricola. Directrices, Dirección General de Agricultura, $48 \mathrm{pp}$.

- (1999b), Evaluación para una mejor gestión. Revisión intermedia de los programas estructurales Objetivos núms. 1 y 6 (1994-1999), Oficina de Publicaciones Oficiales de las Comunidades Europeas, Luxemburgo, 60 pp.

- (1999c), Évaluer les programes socio-economiques, Colección MEANS, Luxemburgo, 6 vols.

- (1999d), Guía para la evaluación del impacto en función del género, Dirección General de Empleo, Relaciones Laborales y Asuntos Sociales, 12 pp. 
COMISIÓN EUROPEA (1999f), Indicadores de seguimiento y evaluación: orientaciones metodológicas, El nuevo período de programación 2000-2006: Documentos de trabajo metodológicos, Documento de Trabajo 3, Dirección General XVI, Política Regional y Cohesión, Coordinación y Evaluación de Operaciones, 67 pp.

- (1999g), La evaluación previa de las intervenciones en el período 2000-2006, El nuevo período de programación 2000-2006: Documentos de trabajo metodológicos, Documento de Trabajo 2, Dirección General XVI, Política Regional y Cohesión, Coordinación y Evaluación de Operaciones, $41 \mathrm{pp}$.

- (1999h), Orientaciones para la evaluación ex-post de los programas del Objetivo 5b. Periodo de programación 1994-1999, Dirección General de Agricultura, 20 pp.

- (1999i), Reforma de los Fondos Estructurales, 2000-2006. Análisis comparado, $31 \mathrm{pp.}$

- (1999j), Reglamento (CE) 1257/1999 del Consejo, de 17 de mayo de 1999, sobre la ayuda al desarrollo rural a cargo del Fondo Europeo de Orientación y de Garantía Agrícola (FEOGA) y por el que se modifican y derogan determinados reglamentos, publicado en el Diario Oficial de las Comunidades Europeas de 26 de junio.

- (1999k), Reglamento (CE) 1260/1999 del Consejo, de 21 de junio de 1999, por el que se establecen disposiciones generales sobre los Fondos Estructurales, publicado en el Diario Oficial de las Comunidades Europeas de 26 de junio.

- (1999l), Reglamento (CE) 1263/1999 del Consejo, de 21 de junio de 1999, relativo al instrumento financiero de orientación de la pesca, publicado en el Diario Oficial de las Comunidades Europeas de 26 de junio.

- (1999m), Reglamento (CE) 1264/1999 del Consejo, de 21 de junio de 1999, que modifica el Reglamento (CE) 1164/1994 por el que se crea el Fondo de Cohesión, publicado en el Diario Oficial de las Comunidades Europeas de 26 de junio.

- (1999n), Reglamento (CE) 1265/1999 del Consejo, de 21 de junio de 1999, que modifica el Anexo II del Reglamento (CE) 1164/1994 por el que se crea el Fondo de Cohesión, publicado en el Diario Oficial de las Comunidades Europeas de 26 de junio.

- (1999p), Reglamento (CE) 1783/1999 del Parlamento Europeo y del Consejo, de 12 de julio de 1999, relativo al Fondo Europeo de Desarrollo Regional, publicado en el Diario Oficial de las Comunidades Europeas de 13 de agosto.

- (1999q), Reglamento (CE) 1784/1999 del Parlamento Europeo y del Consejo, de 12 de julio de 1999, relativo al Fondo Social Europeo, publicado en el Diario Oficial de las Comunidades Europeas de 13 de agosto.

- (1999r), Sexto informe periódico sobre la situación y la evolución socioeconómicas de las regiones de la Unión Europea, Luxemburgo, 242 pp.

- (2000a), Communication from the Commission to the Member States of 28.4.00 laying down guidelines for a Community Initiative concerning economic and social regeneration of cities and of neighbourhoods in crisis in order to promote sustainable urban development. URBAN II, C(2000) 1100, 15 pp.

- (2000b), Comunicación de la Comisión a los Estados miembros de 14.4 .00 por la que se establecen las orientaciones relativas a los Programas de Iniciativas Comunitarias para los que se invita a los Estados miembros a presentar propuestas para la concesión de subvenciones en el marco de la iniciativa EQUAL, C(2000) 1101, 38 pp.

- $(2000 c)$, Comunicación de la Comisión a los Estados miembros de 14.4 .00 por la que se fijan las orientaciones sobre la Iniciativa Comunitaria de desarrollo rural (LEADER+), C(2000) 946, 15 pp.

- (2000d), Comunicación de la Comisión a los Estados miembros de 28.4 .00 por la que se fijan las orientaciones para una iniciativa comunitaria relativa a la cooperación transeuropea para fomentar un desarrollo armonioso y equilibrado del territorio europeo. INTERREG III, C(2000) $1101,38 \mathrm{pp}$.

- (2000e), Evaluation ex-ante et indicateurs pour INTERREG, El nuevo período de programación 2000-2006: Documentos de trabajo metodológicos, Documento de Trabajo 6, Dirección General XVI, Política Regional y Cohesión, Coordinación y Evaluación de Operaciones, $31+6$ pp. 
Comisión Europea (2000f), Las acciones estructurales 2000-2006. Comentarios y reglamentos, Oficina de Publicaciones Oficiales de las Comunidades Europeas, Luxemburgo, 158 pp.

Unión Europea (1992), Tratado de la Unión Europea, firmado en Maastricht el 7 de febrero de 1992.

- (1995), "Mesurer les efects sur l'emploi des interventions structurells communautaires», Cuadernos MEANS, núm. 3, Bruselas, 63 pp.

\section{b) Otras referencias bibliográficas}

AlONSO, Luis Enrique (1994), «Sujeto y discurso: el lugar de la entrevista abierta en las prácticas de la sociología cualitativa», en Juan Manuel Delgado y Juan Gutiérrez (coords.), Métodos y técnicas cualitativas de investigación en ciencias sociales, Madrid, Síntesis, Síntesis Psicología, pp. 225-240.

- (1998), La mirada cualitativa en sociología, Madrid, Fundamentos, 268 pp.

Alvira Martín, Francisco (1983), «Perspectiva cualitativa - perspectiva cuantitativa en la metodología sociológica», REIS, núm. 22, pp. 53-75.

Argyle, Michael (1984), Psicología del comportamiento interpersonal, Madrid, Alianza, Alianza Universidad, $264 \mathrm{pp}$.

Aubel, Judi (1994), Guide pour des études utilisant les discussions de groupe, Ginebra, Bureau International du Travail, $64 \mathrm{pp}$.

BALLART, Xavier (1992), ¿Cómo evaluar programas y servicios públicos? Aproximación sistemática y estudios de caso, Madrid, MAP, $284 \mathrm{pp}$.

BERTRAND, Jane T.; BROWN, Judith E., y WARD, Victoria M. (1992), «Techniques for analyzing focus group data", Evaluation Review, vol. 16, núm. 2, abril, pp. 198-209.

Canales, Manuel, y Peinado, Anselmo (1994), "Grupos de discusión», en Juan Manuel Delgado y Juan Gutiérrez (coords.), Métodos y técnicas cualitativas de investigación en ciencias sociales, Madrid, Síntesis, Síntesis Psicología, pp. 288-316.

Casley, Dennis J., y Kumar, Krishna (1990a), Recopilación, análisis y uso de los datos de seguimiento y evaluación, Madrid, Ediciones Mundi-Prensa/Banco Mundial, 179 pp.

- $(19906)$, Seguimiento y evaluación de proyectos en agricultura, Madrid, Ediciones MundiPrensa/Banco Mundial, 171 pp.

Chambers, Robert (1995), «Los profesionales y los que carecen de poder: ¿Qué realidad importa más?», Opciones, vol. 4, núm. 1, abril, pp. 14-15.

COMUNIDAD DE MADRID (2000), Manual de seguimiento y evaluación de las intervenciones de la Comunidad de Madrid cofinanciadas por los Fondos Europeos, Comunidad de Madrid-Universidad de Alcalá, 191 pp. (mimeo).

CONDE, Fernando (1987), «Una propuesta de uso conjunto de las técnicas cuantitativas y cualitativas en la investigación social. El isomorfismo de las dimensiones topológicas de ambas técnicas», REIS, núm. 39, pp. 213-224.

- (1990), "Un ensayo de articulación de las perspectivas cuantitativa y cualitativa en la investigación social», REIS, núm. 51, pp. 91-117.

Coronado Guerrero, Daniel (1995), «Efectos territoriales de los Fondos Estructurales. Algunas reflexiones sobre los métodos de evaluación aplicados», Boletín Económico del ICE, núm. 2455, pp. 29-39.

Danziger, Sheldon H., y Portney, Kent E. (eds.) (1988), The distributional impacts of public policies, Nueva York, St. Martin’s Press, Policy Studies Organization Series, 257 pp.

Delgado, Juan Manuel, y Gutiérrez, Juan (coords.) (1994), Métodos y técnicas cualitativas de investigación en ciencias sociales, Madrid, Síntesis, 669 pp.

Dunn, Williams N. (1981), Public Policy Analysis. An introduction, Englewood Cliffs, PrenticeHall, 388 pp.

FeInsteIn, Osvaldo N. (ed.) (1994), Experiencias latinoamericanas en seguimiento y evaluación, Quito, IICA-FIDA, 269 pp. 
Feinstein, Osvaldo N. (1995), «To Measure or Not Measure: Comments on Measuring Performance of Agricultural and Rural Development Programs», en R. Piccioto y R. Rist (eds.), Evaluation and development, Washington, D.C., The World Bank.

Hedrick, Terry E. (1994), «The quantitative-qualitative debate: possibilities for integration», New Directions for Program Evaluation, núm. 61, primavera, pp. 45-52.

Hogwood, Brian W., y GunN, Lewis A. (1991), Policy analysis for the real world, Oxford, Oxford University Press, 289 pp.

IBÁÑEZ, Jesús (1985), «Las medidas de la sociedad», REIS, núm. 29, pp. 85-127.

- (1991), El regreso del sujeto. La investigación social de segundo orden, Santiago de Chile, Amerinda Estudios, 201 pp.

- (1992), Más allá de la sociología. El grupo de discusión: técnica y crítica, Madrid, Siglo XXI, 428 pp.

ISLA, María del Mar; Mella, José María, y Soy, Antoni (1998), «La política regional de la UE y su evaluación», en José María MElla MÁrQUeZ, Economía y política regional en España ante la Europa del siglo XXI, Akal Textos, pp. 327-355.

KUMAR, Krishna (1987), Conducting group interviews in developing countries, Washington, Agency for International Development, Program Design and Evaluation Methodology Report, n. ${ }^{\circ}$ 8, 46 pp.

- (1993), "An Overview of Rapid Appraisal Methods in Development Settings", en Krishna Kumar (ed.), Rapid Appraisal Methods, Washington, Banco Mundial, 111 pp.

Mairal, Juan Ángel (1994), La Política de Cohesión Económica y Social en la Unión Europea, Cuadernos de Europa, núm. 7, Zaragoza, Diputación de Zaragoza, 81 pp.

Meny, Ives, y Thoenig, Jean-Claude (1992), Las politicas públicas, Barcelona, Ariel Ciencia Política, $271 \mathrm{pp}$.

Morata, Francesc (1998), La Unión Europea. Procesos, actores y políticas, Barcelona, Ariel, 447 pp.

Morris, Lynn Lyons; Fitz-Gibbon, Carol Taylor, y Freeman, Marie E. (1987), How to communicate evaluation findings, Newbury Park, Sage, Program Evaluation Kit, 92 pp.

Naciones Unidas (1985), Seguimiento y evaluación. Pautas básicas para el diseño y uso de sistemas de seguimiento y evaluación de los proyectos y programas de desarrollo rural en los paises en desarrollo, Roma, Naciones Unidas, $92 \mathrm{pp}$.

OCAMPO, Ada (1994), "Sistematización de la experiencia en seguimiento y evaluación del Proyecto Especial Alto Mayo en Perú», en Osvaldo N. Feinstein (ed.), Experiencias latinoamericanas en seguimiento y evaluación, Quito, IICA-FIDA, pp. 151-205.

PATTON, Michael Quinn (1987), How to use qualitative methods in evaluation, Newbury Park, Sage, Program Evaluation Kit, 176 pp.

SARABIA, Bernabé, y Zarco, Juan (1997), Metodología cualitativa en España, Cuadernos Metodológicos, 22, Madrid, CIS, 127 pp.

Subirats, Joan (1989), Análisis de politicas públicas y eficacia de la Administración, Madrid, MAP, $184 \mathrm{pp}$.

TAYLOR, S. J., y BOGDAN, R. (1992), Introducción a los métodos cualitativos de investigación, Barcelona, Paidós, $343 \mathrm{pp}$.

Uphoff, Norman (1992), Participatory evaluation of rural development projects, Roma, FIDA, División de Seguimiento y Evaluación, 27 pp.

Valadez, Joseph, y Bamberger, Michael (1994), Monitoring and Evaluating Social Programs in Developing Countries, Washington, Banco Mundial.

VIŃAS, Verónica (1994), "Análisis y síntesis comparativa de las lecciones de la experiencia», en Osvaldo N. Feinstein (ed.), Experiencias latinoamericanas en seguimiento y evaluación, Quito, IICA-FIDA, pp. 221-269.

VIN̄AS, Verónica (1995), Los grupos de discusión dirigida en la evaluación de proyectos, Roma, FIDA, División de Seguimiento y Evaluación, 26 pp.

WARD, Victoria M.; BERTRAND, Jane T., y Brown, Lisanne F. (1991), «The comparability of focus group and survey results», Evaluation Review, vol. 15, núm. 2, abril, pp. 266-283. 


\begin{abstract}
The European Structural Funds for regional development policy are in a transition moment. The previous programming ended in 1999, and in the new period the general objective is preserved: economic and social cohesion in an increasingly diversified European Union. Throughout 2000 and 2001 programs have been approved for each European region.

The documentation of the new programming reveals that the European Commission has a growing interest to know the impacts of the activities financed by European Funds. In fact, EU norms corresponding to monitoring and evaluation have experienced important changes between 1999 and 2000: more precision in the references to the necessity and mandatory nature of monitoring and evaluation, more concrete objectives; greater involvement and the responsibilities of the States members; and linking the quality and results of evaluations with financial repercussions for the States or regions. The main emphasis is monitoring and evaluation of structural interventions to know their effectiveness.

Taking into account the evaluations made so far, how to know better the consequences (effects and impacts) on the population and the regions of the policies of regional development?. How can this knowledge be improved? This paper addresses both questions. Two important elements for the quality of the evaluations are: the use of a qualitative approach and the design and use of a system of continuous monitoring of the activities financed with European Funds.
\end{abstract}




\section{TEXTO CLÁSICO}

